\title{
A Síndrome de Burnoute os profissionais de saúde durante a pandemia de Covid-19: uma revisão narrativa
}

\author{
Burnout Syndrome and health professionals during the Covid-19 pandemic: a \\ narrative review
}
Síndrome de Burnout y profesionales de la salud durante la pandemia Covid-19: una revisión narrativa

Vitória Vieira de Sá1*, Letícia Perim de Morais ${ }^{1}$, Lorayne Assis Silva Fernandes ${ }^{1}$, Lupébhia da Silva Nascimento Tarlé ${ }^{1}$, Maressa Precioso Verdin ${ }^{1}$, Maria Letícia de Melo Matos ${ }^{1}$, Mariana Fernandes de Sousa Macêdo ${ }^{1}$, Patrícia Coelho Ferreira ${ }^{1}$, Winicius Marcena Andrade ${ }^{1}$, Maurício Lacerda Caldeira Filho'.

\section{RESUMO}

Objetivo: Discutir a Síndrome de Burnout (SB), caracterizada pelo esgotamento psíquico relacionada à lida laboral no contexto da pandemia de Covid-19. Revisão bibliográfica: A SB é uma doença causada por uma falha no manejo do estresse crônico durante a atividade laboral e representa o maior índice de problema psicossocial. Ela está relacionada a profissões atreladas a valores e compromissos sociais, por esse motivo, o esgotamento mental e insatisfação pessoal podem desencadear Transtorno de Estresse Pós-Traumático, ansiedade, depressão, ideações suicidas e dependência ou abuso de substâncias químicas. Sobre os cuidados individuais voltados à prevenção de SB é necessário ensinar ao profissional técnicas para controlar o estresse, conciliando terapias comportamentais, prática de exercício físico regular, meditação, intensificação do autocuidado preservando o descanso e equilíbrio entre os setores prof issionais e pessoais. Considerações finais: A literatura apontou que os indivíduos expostos, como os profissionais de saúde do enf rentamento ao Covid-19, expostos ao stress e exaustão, estão imersos em um contexto tão extremo que necessitam de oportunidades para recuperar os recursos cognitivos e emocionais esgotados. Desse modo, ficam suscetíveis a desenvolver síndromes psicológicas, como o Burnout.

Palavras-chave: Psiquiatria, Burnout, COVID-19,Trabalhadores.

\section{ABSTRACT}

Objective: To discuss Burnout Syndrome (BS), characterized by mental exhaustion related to work in the context of the Covid-19 pandemic. Bibliographic review: BS is a disease caused by a failure in the management of chronic stress during labor activity and represents the highest rate of psychosocial problems. It is related to professions linked to social values and commitments, for this reason, mental exhaustion and personal dissatisfaction can trigger Post-Traumatic Stress Disorder, anxiety, depression, suicidal ideation and addiction or abuse of chemical substances. Regarding individual care aimed at preventing BS, it is necessary to teach the professional techniques to control stress, combining behavioral therapies, regular physical exercise, meditation, intensifying self-care, preserving rest and balance between the professional and personal sectors. Final considerations: The literature pointed out that exposed individuals, such as health professionals dealing with Covid-19, exposed to stress and exhaustion, are immersed in such an extreme context that they need opportunities to recover depleted cognitive and emotional resources. Thus, they are susceptible to developing psychological syndromes such as Burnout.

Key words: Psychiatry, Burnout, COVID-19, Workers.

1 UNIVAÇO - Instituto Metropolitano de Ensino Superior (IMES), Ipatinga - MG.

*E-mail: vivivieiradesa@hotmail.com

SUBMETIDO EM: 12/2021

ACEITO EM: 12/2021

PUBLICADO EM: 1/2022

REAS | Vol. 15 (1) | DOI: https://doi.org/10.25248/REAS.e9518.2022 


\section{RESUMEN}

Objetivo: Discutir el Síndrome de Burnout (SB), caracterizado por el agotamiento mental relacionado con el trabajo en el contexto de la pandemia Covid-19. Revisión bibliográfica: EI SB es una enfermedad provocada por una falla en el manejo del estrés crónico durante la actividad laboral. y representa la tasa más alta de problemas psicosociales. Se relaciona con profesiones vinculadas a valores y compromisos sociales, por ello, el agotamiento mental y la insatisfacción personal pueden desencadenar Trastorno de Estrés Postraumático, ansiedad, depresión, ideación suicida y adicción o abuso de sustancias químicas. En cuanto a los cuidados individuales orientados a la prevención del SB, es necesario enseñar las técnicas profesionales para el control del estrés, combinando terapias conductuales, ejercicio físico regular, meditación, intensificando el autocuidado, preservando el descanso y el equilibrio entre el sector profesional y el personal. Consideraciones finales: La literatura muestra que las personas expuestas, como los profesionales de la salud que enfrentan Covid-19, expuestos al estrés y al agotamiento, están inmersos en un contexto tan extremo que necesitan oportunidades para recuperar recursos cognitivos y emocionales agotados. Por tanto, son susceptibles de desarrollar síndromes psicológicos como el Burnout.

Palabras clave: Psiquiatria, Burnout, COVID-19, Trabajadores.

\section{INTRODUÇÃO}

A sobrevivência humana e o funcionamento ideal nas situações mais díspares, que requerem um equilíbrio funcional homeostático intrínseco. Nesse contexto, a perturbação desse equilíbrio induzida pelo novo coronavírus, e medidas associadas para conter a sua propagação, irá, inevitavelmente, requerer reajustes para muitos indivíduos no que diz respeito ao funcionamento psicológico e mesmo fisiológico. Destarte, essa ideia é consistente com a hipótese "Reiniciar Função de Equilíbrio" proposta pelo autor (MARKS DF, 2018).

Conforme destacado por Matias T, et al. (2020), as adaptações da Coronavirus Disease 2019 (COVID 19) resultaram em alterações comportamentais, como armazenamento de alimentos e itens essenciais, bem como um aumento da necessidade para autoproteção, junto com contato social reduzido. Logo, durante a pandemia, uma mudança no enfoque humano e na motivação para as necessidades básicas pode explicar as intensas consequências psicológicas observadas. Isso pode incluir mudanças na auto-estima, potencialmente af etadas pelos níveis crescentes de desemprego, pobreza e seu provável impacto no status social.

Sabe-se que a pandemia da SARS-like Coronavirus 2 (SARS-CoV-2) é uma pressão sem precedentes nos serviços de saúde em todo o mundo. Nesse contexto, mudanças consideráveis na prestação de cuidados da saúde necessariamente ocorreram. Por conseguinte, incluíram a cessação dos serviços de rotina, reaproveitamento de áreas clínicas, reafectação de pessoal para ambientes clínicos desconhecidos, e em algumas circunstâncias, o racionamento de serviços. Vale salientar que o impacto dessas condições de trabalho modificadas sobre a cultura da segurança e o bem-estar psicológico são mal compreendidos (DENNING M, et al., 2021).

Ademais, o esgotamento pode ser desencadeado por eventos traumáticos ou condições adversas durante desastres naturais, conflitos e pandemias. Nessa situação, a Síndrome de Burnout (SB), também conhecida como Síndrome do Esgotamento Profissional (SEP), é caracterizada como uma doença psicológica motivada por uma falha no manejo do estresse crônico durante a atividade laboral, que nos últimos 10 anos, ganhou muito espaço nos consultórios médicos e de psicologia. Além do mais, os sintomas mais frequentes e que caracterizam essa o síndrome são falta de energia, distanciamento mental da atividade laboral e, consequente, redução do rendimento profissional (SALVAGIONI DAJ, et al., 2017; RAUDENSKA J, et al., 2019).

Outrossim, surtos de doenças infecciosas têm ef eitos bem documentados sobre o bem -estar psicológico dos profissionais de saúde. Percebe-se que durante a Síndrome Respiratória Aguda Grave (SARS), surtos 
de H1N1 e Ebola, mostraram que os profissionais de saúde da linha de frente estavam em maior risco de desenvolver sequelas psicológicas, incluindo estresse crônico, ansiedade, depressão e Transtorno de Estresse Pós-Traumático (TEPT). Dessa forma, se entende que vários fatores contribuíram para esse fenômeno, como sobrecarga de trabalho, preocupações com exposição ocupacional ou infecção de familiares dos profissionais da saúde. Em comparação com pandemias anteriores, o impacto mental pode ser mais significativo e generalizado, dada a escala dessa pandemia (DENNING M, et al., 2021).

De acordo com Lai J, et al. (2020), os trabalhadores da saúde são responsáveis por prestar os cuidados médicos aos enfermos pela doença, eles se encontram em maior risco de desenvolver o TEPT comparados à população em geral. Portanto, faz-se necessário intervenções no ambiente de trabalho que promovam o bem-estar do indivíduo e reduzam as consequências advindas no esgotamento dos trabalhadores da saúde, como associadas a psicoterapias. Vale destacar, ainda, que os resultados obtidos por Zhang M, et al. (2021) demonstram que a realização de yoga, massagem terapêutica e alongamento são importantes mecanismos físicos, que promovem o relaxamento desses empregados.

Diante ao exposto e considerando a urgência de se abordar a saúde mental dos profissionais de saúde no enfrentamento da pandemia, o presente estudo torna-se de fundamental relevância. Dessarte, objetivouse realizar, por meio de uma revisão narrativa, não apenas uma apresentação de informações acerca da SB, como também discutir e explanar o pico dessa doença ocorrido no período da COVID -19, associado a identificação de formas eficazes para reduzir o estresse ocupacional.

\section{REVISÃO BIBLIOGRÁFICA}

\section{Conceito}

A SB tem origens descritas em desde o século 16, uma vez que a frase "Burn out" já tinha sido mencionada por William Shakespeare. O nome dessa síndrome deriva do termo inglês "burn" queimar e "out" sair. Além de tudo, nas décadas de 1970 e 1980, devido ao aumento do consumo e competitividade, as exigências do mercado de trabalho e desgaste emocional cresceram muito. Sendo assim, a grande quantidade de trabalho e compromissos levam muitas pessoas ao esgotamento, que acarreta até mesmo em depressão (SCHAUFELI WB, 2017).

Segundo o Maslach C, et al. (2001; apud WESTWOOD S, et al., 2017), propôs uma conceitualização da SB em três dimensões distintas: a exaustão, a despersonalização e a relação de ineficácia (redução da realização pessoal). A primeira é caracterizada como o cansaço físico e mental, que é considerada a manifestação mais visível do transtorno e tem associação à jornada de trabalho. Já a segunda é definida como um distanciamento emocional e cognitivo do provedor em relação ao receptor do serviço, isto é, o paciente assume a condição de mero objeto, e com isso o profissional af etado se torna indiferente a ele, o que pode provocar conflitos interpessoais. E, por último, quanto ao terceiro aspecto, a sua relação com os demais é mais complexa, sendo visto como consequência de ambos e apresenta uma ligação com uma demanda excessiva no emprego e falta de recursos.

\section{Etiologia}

A etiologia da doença está relacionada a fatores internos e externos, entre os internos destacam -se os psicológicos, motivados por ambição, extensas horas de trabalho e forte necessidade de reconhecimento. Nessa ótica, entre os fatores externos destacam-se aumento da responsabilidade, ausência de apoio social, restrição administrativa e predomínio de Feedback negativo no ambiente de trabalho. De acordo com alguns as causas para a manifestação da doença são divididas entre baixa realização profissional, despersonalização e exaustão profissional. Desse modo, outros hormônios podem estar envolvidos como serotonina, endorfina, dopamina e ocitocina (RIBEIRO YSFS, et al., 2020).

No que tange à essa síndrome, de acordo com Restauri N e Sheridan AD (2019), diante da crise de recursos associada à pandemia de COVID-19, muitos trabalhadores têm sido vítimas diárias do estresse físico, mental e fobia social. Nesse contexto, a SB tem sido agravada por fato res prejudiciais tais como falta 
de equipamentos de proteção individual, deficiência ou falsidade de informações que chegam com alta velocidade aos pacientes, familiares e profissionais de saúde. Ademais, nesse cenário, a negligência e a imprudência médica por parte dos cuidadores tornam-se mais frequentes, bem como as constantes faltas ao trabalho ou pedidos de demissão, consequências estas que interferem negativamente no combate à pandemia pelo Sars-CoV-2.

Em consonância com Stocchetti N, et al. (2021), os profissionais da área da saúde são mais expostos ao Burnout, pois lida, muitas vezes, com um ambiente de trabalho sem apoio psicossocial, com falta de recursos e uma exigência rotineira para atender as demandas de pacientes infectados. Dessa maneira, ess a conjuntura pode configurar em um começo para o surgimento ou a intensificação do desgaste físico e mental.

No que se trata dos problemas financeiros, da saúde, do "lockdown" e da reorganização laboral exigidos pela COVID-19, muitas pessoas têm relatado insegurança, medo, ansiedade e solidão. Tais fatores têm contribuído para o aumento da incidência desta síndrome. Nesse sentido, é fundamental levar em conta a superlotação dos hospitais brasileiros públicos e privados durante a pandemia e como a SB torno u-se mais frequente entre os profissionais de saúde. Estes, muitas vezes, expostos a jornadas de trabalho antes já extensas, viram sua carga horária de trabalho ainda mais ampliada, fato que contribui para a exaustão física e mental (MOREIRA AH, et al., 2018; DENNING M, et al., 2021).

Todavia, convém ressaltar que para alguns autores, como Vinkers CH e Shaafsma FG (2021), essa patologia ainda é um conceito indescritível, uma vez que não possui um critério para o diagnóstico. Assim, não se estabeleceu uma normativa clínica para avaliar duração e frequência dos sintomas, dessa maneira, a prevalência clínica não pode ser conclusiva.

\section{Impacto}

A pandemia do coronavírus, como pontuam Restauri N e Sheridan AD (2019) e Ribeiro YSFS, et al. (2020), alçou a uma posição de destaque os impactos da SB sobre os profissionais de saúde, a qual teve sua incidência aumentada nesse meio devido ao contexto pandêmico. Além da SEP, a presença constante de estresse enfrentada pelos trabalhadores de saúde predispõem a outros distúrbios psicológicos. Entretanto, vale destacar que nem todos os expostos a estas situações críticas desenvolveram os sintomas. Tal maneira se justifica na resiliência e nos altos níveis de autoconfiança configurarem como fatores protetores para lidar com toda a carga negativa direcionada aos profissionais. Dessa forma, resulta na sensação de controle diante da adversidade e, consequentemente, diminui os prejuízos no ambiente de trabalho e na conjuntura pessoal.

Em consoante aos estudos de Tan BYQ, et al. (2020), indicam que a equipe médica geralmente tem mais capacidade de resiliência do que a equipe não médica. Tal fato se refere na prevalência reduzida, quase que pela metade na equipe médica em comparação com a equipe não médica, de sintomas de $(10,8 \%$ vs $20,7 \%$, respectivamente) e TEPT $(5,7 \%$ vs $10,9 \%)$ em alguns estudos. Vale destacar que vários fatores podem ter af etado os resultados em profissionais de saúde, incluindo o nível de treinamento, experiência ou mesmo no momento em que os entrevistados concluíram a pesquisa em relação ao aumento ou queda de pacientes tratados com COVID.

No que versa à Dantas ESO (2021), em relação aos sentimentos mais comumente apresentados durante a pandemia de COVID-19 podem-se destacar: desesperança, desespero, medo excessivo de recorrência dos fenômenos, temor da morte de si e de pessoas próximas, angústia de ser infectado e de infectar os outros, enfrentamento de medidas de isolamento social, que podem predispor o surgimento de TEPT, sintomas depressivos e ansiosos e de comportamento suicida. Então, em uma pesquisa consistente com uma meta-análise recente de estudos na China e Cingapura, o qual demonstrou que aproximadamente 1 em cada 5 profissionais de saúde experimentou sintomas de ansiedade $(23,2 \%)$ ou depressão (22,8\%) durante a COVID-19 (PAPPA S, et al., 2020).

Em concordância com Denning $M$, et al. (2021), a equipe que foi realocada para novas áreas clínicas teve um risco maior de SB. Isso pode ser devido a condições físicas, como passar longos períodos usando 
equipamentos de proteção ou devido ao estresse de adaptação a um novo ambiente clínico. Nessa situação, as áreas que exigiam pessoal remanejado, por definição, tinham (previam ter) demanda em excesso de recursos, necessitando de realocação de pessoal. Além do mais, a combinação dessas demandas aumentadas, recursos limitados e o estresse psicológico de lidar em um ambiente desconhecido pode ter levado a um aumento nas taxas de esgotamento, esta hipótese seria apoiada pelo modelo de demandasrecursos de burnout.

Diante dessa perspectiva, Lima DS, et al. (2020), Cabello IR e Perez IR (2020), Teixeira FD e Prebianchi HB (2019), apontam que o sofrimento mental, componente importante da SB, impacta tanto em relação ao desempenho laboral do indivíduo, como nas questões econômica e social das instituições de saúde, que atuam de maneira fundamental no contexto da pandemia de COVID-19. Logo, a SB tem repercussões físicas e mentais à saúde dos trabalhadores, os quais têm alterações cardiovasculares, fadiga crônica, cefaléias, enxaqueca, úlcera péptica, insônia, mialgias, artralgias, ansiedade, depressão e irritabilidade.

É mister nos impactos, que a SB, de acordo com Farias MK, et al. (2018), pode afetar na vida pessoal, interf erindo nas relações familiares devido à falta de tempo para o cuidado dos filhos e o lazer, atividades deixadas em segundo plano por causa dos compromissos laborais. Desse modo, o contexto do trabalho é af etado pelo absenteísmo, pela rotatividade de emprego, pelo aumento de condutas violentas e pela diminuição da qualidade do trabalho. Ademais, a SB apresenta-se como um processo progressivo, com aumento da suscetibilidade da doença a partir de 10 anos de trabalho.

\section{Tratamento}

Estudos anteriores sugeriram que burnout é um precursor da depressão, consequentemente, o benefício pode ser visto a partir de intervenções para tratar o esgotamento antes do início da depressão ou ansiedade. No nível individual, as intervenções baseadas em evidências incluem atenção plena, exercício de autoconsciência e entrevistas apreciativas. Já no nível organizacional, os projetos de melhoria da qualidade que melhoram a comunicação organizacional e agilizam os fluxos de trabalho podem reduzir as taxas dessa síndrome (DENNING M, et al., 2021).

Percebe-se que sobre o cuidado com o profissional que está com SB, é necessário acompanhamento psicoterápico, farmacológico e terapias psicossociais (RUIZ-FERNÁNDEZ MD, et al., 2020). Ademais, como demonstrado por Melnyk BM, et al. (2020), na revisão sistemática de 29 estudos, identificou intervenções baseadas na terapia cognitivo-comportamental e de mindfulness, as quais são mais ef etivas na redução do estresse, sintomas ansiosos e depressivos, por consequência, melhora no índice de satisfação com o emprego dos pacientes. Outrossim, McFarland DC, et al. (2019) reforçou o maior impacto positivo de medidas sistêmicas e organizacionais quando comparadas às individuais para a SB.

No que se refere à relação aos cuidados individuais voltados à prevenção da patologia, é necessário ensinar ao colaborador técnicas para controlar o estresse, lançando mão simultaneamente de terapias cognitivo comportamentais, prática de exercício físico regular, meditação e intensificar o autocuidado, uma vez que o descanso e equilíbrio entre os campos profissional e pessoal devem ser preservados. Destarte, no que tange às medidas organizacionais, é de suma importância o desenvolvimento de habilidades interpessoais que melhorem o ambiente laboral, por meio de reestruturação de tarefas, flexibilização de horários e plano de carreira, de modo a atribuir mais qualidade de vida ao trabalhador (PERNICIOTTI P, et al., 2020).

Sabe-se que outras ferramentas válidas para facilitar as consultas relacionadas a problemas de saúde mental incluem recursos de autoavaliação online para rastrear psicopatologia e ajudar a reconhecer os pacientes com maior probabilidade de exigir uma avaliação mais aprofundada. Logo, essa abordagem pode melhorar o gerenciamento do tempo, racionalizar recursos e economizar custos. Estima-se que aproximadamente 10.000 aplicativos "digitais" de saúde mental estejam disponíveis atualmente (MARSHALL JM, et al., 2019).

À face dessa síndrome, as pesquisas que analisam os resultados neuropsiquiátricos diretos e os desfechos indiretos na saúde mental, são fundamentais para melhorar o tratamento, o planejamento dos 
cuidados e a incentivar ações preventivas durante as possíveis pandemias posteriores (VINDEGAARD N e BENROS NE, 2020). Aliás, esses estudos têm como objetivo incentivar a conscientização sobre a importância do rastreamento e do tratamento de doenças psiquiátricas em pacientes com COVID -19 (DENG $J$, et al., 2021).

Dessarte, as atitudes de segurança apresentaram associação significativa com os desfechos psicológicos do estudo. Não era possível inferir se estas atitudes constituíam fatorcontribuinte para burnout, ansiedade e depressão. Todavia, este achado foi muito importante, haja visto que se trata de uma variável modificável e independentemente associada à resolução clínica. Portanto, além de iniciativas que promovam o bem-estar psicológico dos funcionários, a melhoria do ambiente de trabalho, promoção de segurança e atuação em equipe podem ser benéficas e af etar positivamente o prognóstico do quadro (DENNING M, et al., 2021).

\section{Desafios}

As medidas para mitigar os danos recorrentes de sofrimento psicológico após a pandemia de COVID -19 são importantes para prevenir danos a longo prazo. Nesse cenário, foram propostos seis princípios baseados em evidências para apoiar a saúde mental dos profissionais de saúde após a pandemia, que são: avaliação apropriada, investigação de ausências (por motivos de bem-estar), realização de entrevista de retorno ao trabalho, atenção especial aos grupos de risco, monitoramento contínuo da equipe e ajudar os profissionais de saúde a dar sentido à sua experiência (GREENBERG N, et al., 2020; DENNING M, et al., 2021).

No que diz a respeito ao surto deste vírus, criou desafios únicos para os assalariados em todo o mundo. Moura EC, et al. (2020) apontou que, enquanto a modalidade home office de trabalho foi usada como ferramenta de controle dos níveis de contaminação em muitas áreas de atuação, na área da saúde, os profissionais foram cada vez mais expostos a esse risco por estarem na linha de frente do combate a pandemia de COVID-19. Além disso, as jornadas de trabalho aumentaram e as condições de trabalho pioraram devido à falta de leitos, equipamentos de proteção individual e treinamento.

É válido salientar que por ser a Sars-CoV-2 altamente transmissível, alguns fatores relacionados ao risco de contágio no ambiente de trabalho e a adoção de procedimentos preventivos podem causar diversas preocupações do campo mental. Por exemplo, a falta de equipamentos de proteção individual, o peso físico provocado pelo uso, o medo de se infectar e de que isso possa prejudicar os familiares, o conflito entre os procedimentos de segurança e o desejo de dar suporte, jornada de trabalho mais longa, pressão a multitarefa e a estigmatização de pessoas que trabalham em ambientes de alto risco podem afetar profundamente o bem-estar mental dos empregados (GIORGI G, et al., 2020).

Conforme Schwartz R, et al. (2020), a pandemia da COVID-19 apresenta desafios sem precedentes devido à incerteza prolongada e ansiedade elevada, ameaça imediata à segurança pessoal e familiar, isolamento social, testemunha de sofrimento físico e morte e demandas profissionais em evolução. D esse modo, esses estressores individuais se combinam para induzir hiperexcitação, hipervigilância, distúrbios do sono, pensamentos intrusivos, depressão e luto.

A SB entre os trabalhadores da saúde já era um problema significativo há anos. De acordo com West $\mathrm{CP}$, et al. (2018) estimou que, à época, a prevalência de sinais e sintomas característicos da síndrome era próxima de $50 \%$ nos Estados Unidos, e mesmo na ausência de dados globais, estimava-se taxas similares em outros países. Aliás, segundo o Yates SW (2020) salientou o impacto do estresse gerado pela tecnologia da informação, sobretudo de sistemas de prontuário eletrônico (EMR), com que eles têm dificuldades em utilizar devido ao excesso de necessidade de informações, interface ineficiente, tempos de resposta imprevisíveis e falta de integração com outros sistemas, e há uma associação entre maior número de horas gastas com o EMR e essa síndrome em profissionais da atenção primária e psiquiatras.

Não obstante, a médio e longo prazo, os efeitos psicológicos da COVID-19 sejam atualmente desconhecidos, tendo em vista as taxas de prevalência detectadas nos estudos e na fase aguda, é aconselhável que os sistemas de saúde estejam proporcionalmente prep arados para atender aos possíveis 
aumento da demanda por serviços de saúde mental. Assim, com base nas descobertas durante os estágios iniciais da pandemia, há uma necessidade clara de identificar as consequências psicológicas de longo prazo, a fim de garantir que haja uma of erta adequada de serviços (ARORA T, et al., 2020).

\section{CONSIDERAÇÕES FINAIS}

Diante dessa conjuntura, percebe-se que o esgotamento profissional epsicológico são fruto da síndrome de Burnout. Esta doença está cada vez mais presente entre os profissionais de todas as áreas, especialmente entre os trabalhadores da área da saúde, que tiveram de lidar com a superlotação dos hospitais, o manejo de casos clínicos cada vez mais complicados, muitas mortes e o medo de contaminar as pessoas que amam por lidarem com o vírus diariamente. Além disso, é importante ressaltar que a SB envolve não só o indivíduo acometido, mas altera toda uma dinâmica do ambiente laboral, familiar e social do paciente, tornando-se um problema de saúde pública. Perante a essa situação, é essencial fortalecer a importância do autocuidado, através de psicoterapia e outras técnicas eficazes para amenizar os sintomas decorrentes aflorados nestes tempos.

\section{REFERÊNCIAS}

1. ARORA T, et al. The prevalence of psychological consequences of COVID-19: a systematic review and metaanalysis of observational studies. Journal of Health Psychology, 2020;1:1-20.

2. CABELLO IR, PEREZ IR. El impacto de la pandemia por COVID-19 sobre la salud mental de los profesionales sanitarios. Escuela Andaluza de Salud Pública: Consejería de Salud y Familias, 2020.

3. DANTAS ESO. Saúde mental dos profissionais de saúde no Brasil no contexto da pandemia por Covid-19. Interface, 2021;25: e200203.

4. DENG J, et al., The prevalence of depression, anxiety, and sleep disturbances in COVID-19 patients: a metaanalysis. Annals of New York Academy of Sciences, 2021;1486: $90-111$.

5. DENNING M, et al. Determinants of burnout and other aspects of psychological well-being in healthcare workers during the Covid-19 pandemic: a multinational cross-sectional study. PLoS One, 2021; 16: e0238666.

6. FARIAS MK, et al. As consequências da síndrome de burnout em profissionais de enfermagem: revisão integrativa. Caderno de Graduação - Ciências Biológicas e da Saúde (UNIT), 2018; 4: 259-270.

7. GIORGI G, et al. COVID-19-related mental health effects in the workplace: a narrative review. International Joumal of Environmental Research and Public Health, 2020;17: 7857.

8. GREENBERG N, et al. Managing mental health challenges faced by healthcare workers during Covid-19 pandemic. BMJ, 2020;368: $\mathrm{m} 1211$.

9. LAI J, et al. Factors associated with mental health outcomes among health care workers exposed to coronavinus disease 2019. JAMA Network Open, 2020;3: e203976.

10. LIMA DS, et al. Recommendations for emergency surgery during the COVID-19 pandemic. Journal of Health \& Biological Sciences, 2020; 8: 1-3.

11. MARKS DF. A reset for psychology as natural science. The Psychologist, 2018. Disponível em: https://thepsychologist.bps.org.uk/reset-psychology-natural-science. Acesso em:29 de nov. de 2021.

12. MASLACH C, et al. Job burnout. Annual Review of Psychology, 2001; 52:397-422. Apud: WESTWOOD S, et al. Predictors of emotional exhaustion, disengagement and burnout among improving access to psychological therapies (IAPT) practitioners. Journal of Mental Health, 2017;26:172-179.

13. MARSHALL JM, et al. The digital psychiatrist: in search of evidence-based apps for anxiety and depression. Frontiers in Psychiatry, 2019; 10: 1-7.

14. MATIAS T, et al. Human needs in COVID-19 isolation. Journal of Health Psychology, 2020;25: 871-882.

15. MCFARLAND DC, et al. Update on addressing mental health and burnout in physicians: what is the role for psychiatry? Current Psychiatry Reports, 2019;21: 108.

16. MELNYK BM, et al. Interventions to improve mental health, well-being, physical health, and lifestyle behaviors in physicians and nurses: a systematic review. American Journal of Health Promotion, 2020;34:929-941.

17. MOREIRA AH, et al. Síndrome de Burnout em médicos: uma revisão sistemática. Revista Brasileira de Saúde Ocupacional. Brasil, 2018;43: 1-11.

18. MOURA EC, et al. Epidemia de burnout durante a pandemia de Covid-19: O papel da LMX na redução do burnout dos médicos. Revista de Administração de Empresas, 2020;60:426-436.

19. PAPPA S, et al. Prevalence of depression, anxiety, and insomnia among healthcare workers during the COVID-19 pandemic: A systematic review and meta-analysis. Brain, Behavior and Immunity, 2020;88: 901-907.

20. PERNICIOTTI P, et al. Síndrome de Burnout nos profissionais de saúde: atualização sobre definições, fatores de risco e estratégias de prevenção. Sociedade Brasileira de Psicologia Hospitalar, 2020;23: 35-52.

21. RAUDENSKA J, et al. Occupational burnout syndrome and post-traumatic stress among healthcare professionals during the novel coronavirus disease 2019 (COVID-19) pandemic. Best Practice \& Research Clinical Anaesthesiology, 2020;34:553-560. 
22. RESTAURI N, SHERIDAN AD. Burnout and posttraumatic stress disorder in the coronavirus disease 2019 (COVID19) pandemic: intersection, impact, and interventions. Journal of the American College of Radiology, 2020;17:921 926.

23. RIBEIRO YSFS, et al. Implicações da Síndrome de Burnout em profissionais da saúde durante a pandemia de COVID-19: Uma revisão integrativa. Revista Científica Multidisciplinar Núcleo do Conhecimento, 2020;10: 87-107.

24. RUIZ-FERNÁNDEZ MD, et al. Compassion fatigue, burnout, compassion satisfaction and perceived stress in healthcare professionals during the COVID-19 health crisis in Spain. Journal of Clinical Nursing, 2020; 29: 43214330.

25. SALVAGIONI DAJ, et al. Physical, psychological and occupational consequences of job burnout: A systematic review of prospective studies. PLoS One, 2017;12: e0185781.

26. SCHWARTZ R, et al. Addressing post pandemic clinician mental health: a narrative review and conceptual framework. Annals of Internal Medicine, 2020;173:981-988.

27. SCHAUFELI WB. Burnout: a short socio-cultural history. In: NECKEL S, et al. (Eds.). Burnout, fatigue and exhaustion: an interdisciplinary perspective on a modern affliction. Londres: Palgrave Macmillan, 2017;316p.

28. STOCCHETTI N, et al. Burnout in Intensive Care Unit Workers during the Second Wave of the COVID-19 Pandemic. International Journal of Environmental Research and Public Health, 2021; 18:6102.

29. TAN BYQ, et al. Psychological impact of the COVID-19 pandemic on health care workers in Singapore. Annals of Internal Medicine, 2020;173: 317-320.

30. TEIXEIRA FD, PREBIANCHI HB. Comprometimento, estresse e satisfação com a vida de profissionais da saúde. Revista Psicologia Organizações e Trabalho, 2019;15: 598-606.

31. VINDEGAARD N, BENROS ME. COVID-19 pandemic and mental health consequences: systematic review of the current evidence. Brain, Behavior, and Immunity, 2020;89:531-542.

32. VINKERS CH, SHAAFSMA FG. Burnout urgently needs robust Research. Nature, 2021;591:489-491.

33. ZHANG M, et al. Physical relaxation for occupational stress in healthcare workers: A systematic review and network meta-analysis of randomized controlled trials. Journal of Occupational Health, 2021. 